function for the $\mathrm{Mg}^{2+}$-activated enzyme which acquires calcium sensitivity at a relatively late stage in development, for example, by interaction with 'coupling factors' which link ATP hydrolysis to $\mathrm{Ca}^{2+}$ transport. The lack of a subunit structure for the $\mathrm{Ca}^{2+}$ activated ATPase of rabbit sarcoplasmic reticulum tends to make this assumption less likely, although it is an intriguing possibility.

\section{Solar wind blows gusty at sunspot maximum}

by our Cosmology Correspondent

THE continuing story of the influence of the Sun on terrestrial events has developed a stage further with the report by Intriligator of observations which provide the first evidence for long term variations in the solar wind associated with changes in the solar cycle (Astrophys. J. Lett., 188, L23; 1974). Events such as weather variations and modulation of the Earth's spin rate associated with the solar cycle of activity are now becoming well documented, and in recent years it has been established that there is a solar modulation of cosmic rays which is in antiphase with the solar cycle. All this evidence points to variations in the solar wind as, if not the root cause, then at least the next link in the causal chain. But since suitable space vehicles which can provide essentially continuous monitoring of the solar wind have only been available for some ten yearsabout the length of one solar cycle-it is hardly surprising that such solar wind variations have only now been directly confirmed.

Intriligator's work is concerned with variations in the solar wind which are the result of changes in the high speed stream structure. The data were obtained by Pioneer and Vela spacecraft; they show variations in the number and intensity of high speed proton streams, and the average solar wind speed, between 1965 and 1971. Early in the period studied a high solar

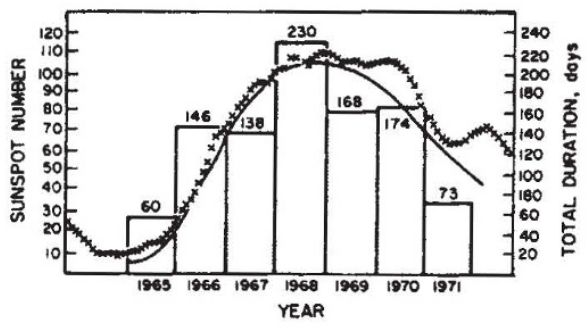

Total number of days on which high speed "gusts" were observed in the solar wind. —, Smoothed sunspot numbers; - $\mathrm{x}$-, measured sunspot numbers. Data for 1965 and 1971 are based on 136 and 270 days of data, respectively. wind speed of $626 \mathrm{~km} \mathrm{~s}^{-1}$ was measured, on January 20,1966, and in all five high speed streams (the slowest with velocity $497 \mathrm{~km} \mathrm{~s}^{-1}$ ) were observed between December 25, 1965 and February 3, 1966. But this represented the low point of activity during the period of the investigation.

When longer stretches of data are examined, it turns out that there were more high speed streams in 1968 (44) than in any of the other years of the study; as Intriligator puts it, the solar wind is more "gusty" around the time of maximum solar activity. On 230 days in 1968 there was high speed streaming activity in the solar wind; at the other extreme, all but 73 days out of 270 studied in 1971 were free from gusts.

This immediately offers an explanation of the modulation of cosmic rays over the solar cycle. At solar maximum, there is a much greater chance that cosmic rays entering the Solar System will encounter high speed streams, and this produces more modulation of the cosmic rays. When the solar wind is calm, it is easier for cosmic rays to penetrate the solar wind and reach Earth. The yearly average of the solar wind speed itself varies over the solar cycle, and is highest at solar maximum; there seems little doubt that this is a real effect which provides an important insight into the workings of the Solar System.

\section{Genome organisation in eukaryotes}

from a Correspondent

ANALYSIS of the organisation of nucleotide sequences in DNA of the more highly evolved eukaryotes is proceeding rapidly at present and has revealed some intriguing but so far unexplained phenomena. Following up their initial observations on the formation of DNA rings and other circular structures ( $J$. molec. Biol., 51, 621; 1970), Thomas and his associates in a series of papers (ibid., 77, 25-99; 1973) have estimated that as much as half the DNA in mammals, insects and amphibians is composed of tandemly repeated or intermittent tandemly repeated sequences clustered in groups which they call $g$ regions. The number of $g$ regions in three Drosophila species is about 5,000 which corresponds to the number of cytologically observable bands or chromomeres in their polytene chromosomes.

Similar measurements for the mouse and Necturus, which have respectively fifteen and seven hundred times more DNA than Drosophila, reveals that the number of $g$ regions is about 40,000 and 1.5 million respectively. This probably means that the one to one correlation between $g$ region and chromomere number observed in Drosophila does not hold in other organisms since the chromomere number (lampbrush chromosome loops) in Triturus has been estimated at 4,000. These results have relevance for the problem of the very large differences in genome size between closely related organisms. Increased genome sizes seem to be based largely on increased numbers and only marginally on increased size of $g$ regions, since the estimated sizes of $g$ regions in mouse and Necturus are only twice that of Drosophila.

The kinds of experiments Thomas and his colleagues have done to reach these conclusions are the measurement of the frequency and contour length of rings and ringlike stuctures observed by electron microscopy in preparations of DNA renatured to low $C_{o} t$ values after treatment with Escherichia coli exonuclease III, an enzyme which digests one strand of a DNA duplex from $3^{\prime}$ to $5^{\prime}$. DNA molecules resected by this enzyme will have two single strand ends which if complementary will tend to renature to give rings and other ring-like structures. No rings are produced by treatment of bacterial DNA in the same way, though many phages may cyclise through the presence of so called sticky ends which may occur naturally as in $\lambda$ or produced by exonuclease III treatment as in $\mathrm{T} 7$.

Thomas et al. have shown that the frequency of rings is linearly related to the extent of resection up to a maximum plateau level which is 300 nucleotides for mouse and Drosophila and 500 for Necturus. These figures are presumably related to the length of the repeating sequences involved. Providing rings are formed in optimal resection and renaturation conditions, the frequency of rings is primarily a function of DNA size and is maximum with DNA of $1.2 \mu \mathrm{m}$ in length regardless of organism. Such rings have very good thermal stability suggesting that the region of overlap is of the order of 200 nucleotides with very little if any mismatching, since thermal stability under ionic conditions reffects both length and fidelity of hybrid duplex. This means that the minimum length of the repeat unit is 200 nucleotides and that they show very little divergence by base substitution.

Fewer rings are created both with larger and with smaller DNA molecules. The reduction with increasing length is interpreted to mean that related repeated sequences are clustered into regions which Thomas et al. call $g$ regions. An alternative explanation for this observation-that longer fragments have a greater distance separating potentially reactive single strand ends resulting in slower renaturation kinetics -was eliminated by a study of the effect of fragment length on the rate of 